\title{
Control of Chaos in Combustion Reactions
}

\author{
M. L. Davies, P. A. Halford-Maw, J. Hill, M. R. Tinsley, B. R. Johnson, and S. K. Scott* \\ School of Chemistry, University of Leeds, Leeds LS2 9JT, U.K.
}

\section{Z. Kiss and V. Gáspár}

Institute of Physical Chemistry, University of Debrecen, P.O. Box 7, H-4010 Debrecen, Hungary

Received: June 12, 2000; In Final Form: August 23, 2000

\begin{abstract}
The chaotic evolution in the combustion of $\mathrm{CO}$ in a well-stirred flow reactor is controlled experimentally using a modified form of the simple proportional feedback (SPF) algorithm. An unstable period-1 oscillation is stabilized through the imposition of small, appropriate perturbations which are calculated from the observed experimental response of the system and do not require any information concerning the reaction mechanism. It is observed that the algorithm is significantly more efficient if these perturbations are applied for only a fraction of the oscillatory period. A similar observation is made from a numerical study of a model for the $\mathrm{H}_{2}+\mathrm{O}_{2}$ reaction, and it is shown that this arises because the perturbations shift the system significantly from the attractor of the unperturbed system. The duration of the perturbation in each cycle then becomes a second control parameter and effects a higher-dimensional control algorithm in a simple manner appropriate to experimental implementation for such demanding systems. The control strategy is seen to be sufficiently robust to operate even though the system shows a marked drift over the course of the experiment. Some comments concerning strategies for the optimal implementation of SPF methods are then made.
\end{abstract}

\section{Introduction}

The spontaneous oxidation reaction of $\mathrm{CO}$ provides one of the simplest combustion systems and has great technical importance, representing the final stage of oxidation of all hydrocarbon fuels to $\mathrm{CO}_{2}$ with the associated heat release. Traditional studies involving closed reaction vessels have established the existence of a pressure-temperature $\left(p-T_{\mathrm{a}}\right)$ ignition limit peninsula at subatmospheric pressures similar to that for the $\mathrm{H}_{2}+\mathrm{O}_{2}$ reaction system. ${ }^{1}$ The $\mathrm{CO}+\mathrm{O}_{2}$ reaction is also able to support oscillatory behavior (oscillatory glow or the "lighthouse effect") in closed systems, ${ }^{2,3}$ although this response is extremely sensitive to the presence of hydrogencontaining impurities (such as $\mathrm{H}_{2} \mathrm{O}$ or $\mathrm{CH}_{4}$ ) and to the state of the reactor surface. ${ }^{4,5}$ In an extended series of papers, the Leeds group has established that this reaction can be studied in a wellstirred flow reactor (CSTR) and there also exhibits an ignition limit. ${ }^{6}$ For ambient temperatures above this ignition limit, the reaction typically exhibits an oscillatory ignition mode. This is highly reproducible provided careful attention is paid to repeatable experimental technique. The sensitivity of the reaction to impurities can be tamed by introducing a known concentration of $\mathrm{H}_{2}$ into the $\mathrm{CO}$ feed at some level above any impurity concentration. Johnson and $\mathrm{Scott}^{7}$ have shown that complex oscillations arise in this system over a range of operating conditions and have characterized these as arising through a classic period-doubling cascade, leading to deterministic chaos. ${ }^{8}$ The chaotic behavior supports a classic, single-humped nextmaximum map.

There has been much recent interest in controlling systems that exhibit deterministic chaos, i.e., in stabilizing different periodic responses under conditions for which the uncontrolled system exhibits a chaotic response through the imposition of appropriate small-amplitude perturbations to the operating conditions without exiting the region of the chaotic response. The initial theoretical suggestion of Ott, Grebogi, and Yorke ${ }^{9}$ was rapidly cast in a form particularly suited to experimental implementation by Peng, Petrov, and Showalter ${ }^{10}$ in terms of a simple proportional feedback (SPF) algorithm. This approach has been successfully applied to a number of experimental systems, including the solution-phase Belousov-Zhabotinsky (BZ) reaction. ${ }^{11}$ Other approaches, including the Pyragas method ${ }^{12}$ and the OPF method, ${ }^{13}$ have been exploited for the BZ system, ${ }^{14}$ enzyme reactions, ${ }^{15}$ and electrochemical systems. ${ }^{16-19}$ For a full survey, see refs 20 and 21.

In this paper we explore the application of the SPF method to control of the chaotic combustion in the $\mathrm{CO}+\mathrm{O}_{2}$ system. Several "experimental difficulties" are presented, and we discuss their resolution. In particular, we determine the optimum form of the control perturbation for this type of system in terms of a modified SPF algorithm in which the perturbations are applied only for a fraction of the period. These aspects are further confirmed numerically through the control of chaos in a model of the $\mathrm{H}_{2}+\mathrm{O}_{2}$ system, which also exhibits chaotic ignition behavior in a CSTR and for which a satisfactory kinetic mechanism has been established. ${ }^{22}$ We conclude with some general comments about the operation of the SPF method in practice and the limits on its successful implementation.

\section{Experimental Section}

The full details of the basic experimental apparatus and procedure have been published elsewhere. ${ }^{7,8}$ Briefly, the reactor comprises a $0.57 \mathrm{dm}^{3}$ spherical Pyrex vessel mounted in a recirculating-air furnace providing temperature control temporally $( \pm 0.5 \mathrm{~K})$ with spatial uniformity $( \pm 1 \mathrm{~K})$ across the reactor surface. Reactant-grade gases are supplied from cylinders of $\mathrm{O}_{2}$ and of a $\left(\mathrm{CO}+1 \% \mathrm{H}_{2}\right)$ mixture, subject to separate 
preheating, through individual electronic mass flow controllers. The pressure is set by a needle valve, and the inflow rates are adjusted to provide the desired mixture composition and mean residence time. The reaction is monitored by a photomultiplier tube (PMT) to monitor emission (there is chemiluminesence associated with the formation of electronically excited $\mathrm{CO}_{2}$ during ignition). New features relevant to the present study include computer control of the voltage supply to the mass flow controllers and data acquisition of the output signal from the PMT to the controlling PC with a sampling rate of $1-10^{4} \mathrm{~s}^{-1}$. The output from the PMT is also recorded on a chart recorder.

The control experiments in this work are operated at a total pressure in the reactor of $19 \mathrm{mmHg}(2.53 \mathrm{kPa})$ with a mean residence time $t_{\text {res }}=25 \pm 3 \mathrm{~s}$ and an unperturbed mixture composition with $\left(\mathrm{CO}+1 \% \mathrm{H}_{2}\right): \mathrm{O}_{2}=7.1: 5.6$ (approximately 1.3:1). The response of the system operating under these conditions to changes in the ambient temperature is determined by increasing $T_{\mathrm{a}}$ in small steps and allowing the system to settle fully before the output from the thermocouple is recorded. In this way, the location of the $p-T_{\mathrm{a}}$ ignition limit and subsequent changes in oscillatory waveform can be mapped out. Hysteresis effects can be determined by locating the boundaries as $T_{\mathrm{a}}$ is subsequently reduced.

The SPF control algorithm requires the determination of the next-maximum map during chaotic operation and then the identification of the "fixed point" of that map, the slope of the map in the vicinity of the fixed point, and, finally, the shift in the map arising from a small perturbation to one of the operating parameters. In this work, the perturbation is applied to the mass flow rate of the $\mathrm{CO}+1 \% \mathrm{H}_{2}$ inflow.

The procedure for implementation of the SPF algorithm here is as follows. The output from the PMT is somewhat noisy due to background glow from the heating coils and other "stray light" sources. In principle, the light could be filtered to collect only the chemiluminescence at $420 \mathrm{~nm}$, but this weak emission is not sufficiently strong to provide a useful signal alone. It is important to be able to distinguish between the peak light intensity during an ignition from smaller maxima due to noise, so a "threshold" signal is selected and data are only collected when the emission intensity exceeds this value. Once the ambient temperature has been adjusted such that the system lies within the chaotic regime and has stabilized sufficiently, a "baseline signal" from the PMT is established by monitoring the voltage recorded on the analog-to-digital converter between peaks over a period of several ignitions (typically this will have a value of the order of $0.2 \mathrm{mV}$ ). A threshold signal, typically exceeding the baseline by $0.2 \mathrm{mV}$, is then selected. The baseline is sampled at random during the subsequent evolution of the system, and the threshold is adjusted dynamically to remain at this increment above the baseline.

In the "data collection" mode, the output signal from the PMT is monitored, and if this increases beyond the selected threshold value, the sampling rate is increased appropriately and the voltage values are captured into the PC memory and displayed in an appropriate window on screen. Each value is compared with the previous value, and the previous value is discarded if it is lower. The maximum emission intensity during the ignition event determined in this way is recorded. The sampling rate is reduced when the signal falls below the threshold. This procedure repeats at the next crossing of the threshold, so the maximum emission intensity for the next ignition is recorded and so on. The maximum for one ignition is plotted against the maximum for the next ignition (the next-maximum map) in a separate window.

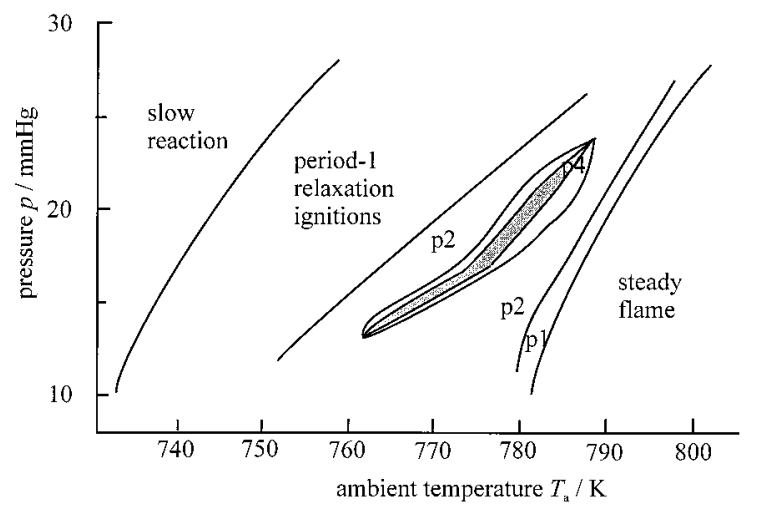

Figure 1. Pressure-ambient temperature $\left(p-T_{\mathrm{a}}\right)$ ignition limit diagram for system with $\left(\mathrm{CO}+1 \% \mathrm{H}_{2}\right): \mathrm{O}_{2}=7.2: 5$.6 showing region of chaotic evolution arising from period-doubling cascades.

TABLE 1: Extent of Chaotic Region for System with $p=19$ mmHg

\begin{tabular}{lccc}
\hline \multicolumn{1}{c}{ parameter } & lower boundary & upper boundary & value used \\
\hline temperature $^{a}(\mathrm{~K})$ & $786( \pm 2)$ & $791( \pm 2)$ & 789 \\
$\mathrm{O}_{2}$ flow $^{b}(\mathrm{sccm})$ & $4.0( \pm 0.1)$ & $9.0( \pm 0.15)$ & 5.6 \\
$\mathrm{CO} \mathrm{flow}^{c}(\mathrm{sccm})$ & $6.9( \pm 0.5)$ & $7.4( \pm 0.2)$ & 7.14
\end{tabular}

${ }^{a} \mathrm{sccm}=$ standard cubic centimeters per minute; $w i t h f_{\mathrm{O}_{2}}=5.6 \mathrm{sccm}$ and $f_{\mathrm{CO}}=7.14 \mathrm{sccm} .{ }^{b}$ With $T=789 \mathrm{~K}$ and $f_{\mathrm{CO}}=7.14 \mathrm{sccm} .{ }^{c}$ With $T=789 \mathrm{~K}$ and $f_{\mathrm{O}_{2}}=5.6 \mathrm{sccm}$.

Once sufficient points on the next-maximum map have been collected, a small change is made to the voltage supplied by the PC to the fuel mass flow controller and the above procedure is repeated to determine the new map. Once the two maps have been collected, the data are analyzed to provide numerical estimates of the fixed point and slope of the map in the vicinity of the fixed point, for both the unperturbed system and the perturbed system. This gives an estimate of the appropriate control parameters for the SPF algorithm. In practice, the user may need to "refine" the values of these control parameters, which typically have a high uncertainty, when the algorithm is implemented (see below).

\section{Results}

(a) $\boldsymbol{p}-\boldsymbol{T}_{\mathrm{a}}$ Ignition Limit Diagram. The mixture composition and mean residence time employed in this study differ slightly from that used by Johnson and Scott, so the first task is to determine the experimental conditions for the various dynamic regimes. The so-called $p-T_{\mathrm{a}}$ ignition limit diagram for this present system, with an mass flow rate of $\mathrm{O}_{2} f_{\mathrm{O}_{2}}=5.6$ standard cubic centimeter per minute $(\mathrm{sccm})$ and a mass flow rate of $\mathrm{CO} f_{\mathrm{CO}}=7.2 \mathrm{sccm}$, is shown in Figure 1. For ambient temperatures above the ignition limit there are various regions corresponding to different periodic states, with boundaries corresponding to period-doubling bifurcations. There is also a distinguished region for which the reaction exhibits an aperiodic (chaotic) response. This region can be entered by increasing (or decreasing) the ambient temperature or pressure, as indicated from Figure 1, and also by varying the flow rates of the individual reactants (changing the mixture composition and mean residence time). The extent of the region of chaotic behavior in terms of the experimental parameters that can be varied conveniently during an experiment (and hence are potential control parameters) is given in Table 1 .

Thus, the chaotic region is narrow in terms of the ambient temperature, but reasonably wide for the $\mathrm{O}_{2}$ and $\mathrm{CO}$ inflow rates (in terms of the degree of experimental control the operator has over these quantities), with chaos existing over the range from 


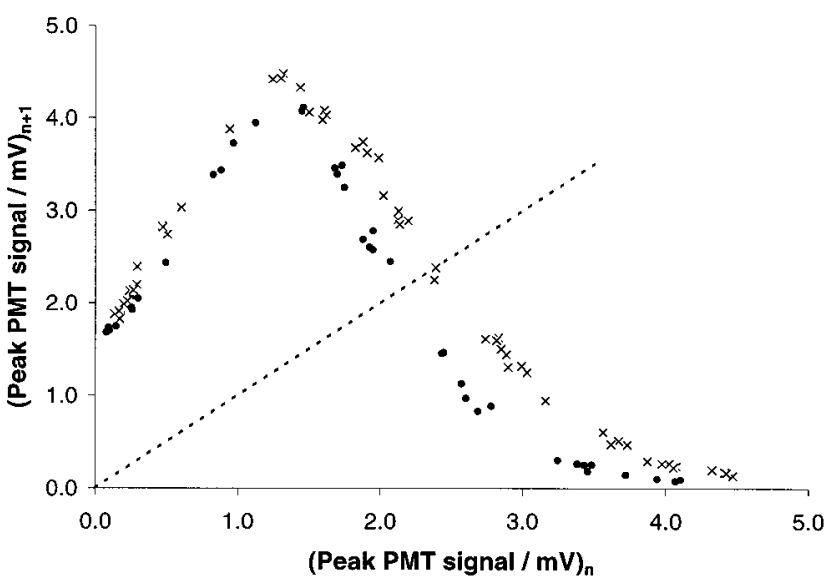

Figure 2. Next-maximum temperature maps for two different parameter values within chaotic region: $\times, f_{\mathrm{CO}}=7.14 \mathrm{sccm} ; \bullet, f_{\mathrm{CO}}=7.41$ $\mathrm{sccm}$. The dashed line $x_{n+1}=x_{n}$ is shown to help indicate the location of the unstable period-1 fixed point.

4.0 to $9.0 \mathrm{sccm}$ in the former case and from 6.9 to 7.4 in the latter. The system was found to be more responsive to variations in the CO flow, so this was chosen as the control parameter for the present study. Throughout this paper, then, we take $T_{\mathrm{a}}=$ $789 \mathrm{~K}$, an $\mathrm{O}_{2}$ flow rate of $5.6 \mathrm{sccm}$, and, for the unperturbed system, take a CO flow rate $f_{\mathrm{CO}}=7.14 \mathrm{sccm}$.

(b) Next-Maximum Maps. Using the procedure described in section 2, the next-maximum map has been determined for a system operating at the above conditions with a $\mathrm{CO}$ inflow rate $f_{\mathrm{CO}}=7.14 \mathrm{sccm}$ and again for a "perturbed system" with $f_{\mathrm{CO}}=7.41 \mathrm{sccm}$ (note that this is the maximum $\mathrm{CO}$ flow rate for which chaotic evolution is observed under these conditions). These maps are displayed in Figure 2. The period-1 fixed point is identified by the intersection of the map with the identity line $x_{n+1}=x_{n}$ and the slope of the map and the shift in the fixed point is determined from these data (see below).

(c) SPF Control. The light emission intensity exhibits a classic single-humped maximum, as illustrated in Figure 2, suggesting that the SPF control method will be appropriate for chaos control. If we represent the map in the form

$$
x_{n+1}(f)=F\left(x_{n} f\right)
$$

where $x_{n}$ and $x_{n+1}$ are the current and next maximum values and $f$ is the $\mathrm{CO}$ flow rate, then linearizing in the vicinity of the fixed point $x_{\mathrm{F}}$, we can write

$$
x_{n+1}(f)=m\left[x_{n}-x_{\mathrm{F}}(f)\right]+x_{\mathrm{F}}(f)
$$

where $m$ is the slope of the map at the fixed point $x_{\mathrm{F}}$. If the flow rate is varied by a small amount $\delta f$ and if this variation moves the fixed point without significantly changing the gradient $m$, the linearized map can be written as

$$
x_{n+1}(f+\delta f)=m\left[x_{n}-x_{\mathrm{F}}(f+\delta f)\right]+x_{\mathrm{F}}(f+\delta f)
$$

where

$$
x_{\mathrm{F}}(f+\delta f)=\delta f \frac{\mathrm{d} x_{\mathrm{F}}(f)}{\mathrm{d} f}+x_{\mathrm{F}}(f)
$$

If during the evolution of the unperturbed system (with flow rate $f$ ) we observe a point on the map close to that corresponding to the unstable period-1 fixed point, so that

$$
x_{n}=x_{\mathrm{F}}(f)+\Delta x
$$

where $\Delta x \ll x_{\mathrm{F}}$, then a perturbation $\delta f$ to the flow, such that

$$
x_{n+1}(f+\delta f)=x_{\mathrm{F}}(f)
$$

will direct the system to the (unstable) fixed point of the unperturbed system. The necessary perturbation ${ }^{10}$ is given by

$$
\delta f=\frac{m}{(m-1) \mathrm{d} x_{\mathrm{F}} / \mathrm{d} f} \Delta x \equiv \frac{\Delta x}{g}
$$

where the "control parameter" $g$ is defined as

$$
g=\frac{(m-1)}{m} \frac{\mathrm{d} x_{\mathrm{F}}}{\mathrm{d} f}
$$

The value of $g$ can be determined, therefore, from the experimental next-maximum maps for the perturbed and unperturbed systems such as those shown in Figure 2. For those data, we obtain the following values:

unperturbed system: $f_{\mathrm{CO}}=7.14( \pm 2 \%) \mathrm{sccm}$,

$$
\text { fixed point } \begin{aligned}
x_{\mathrm{s}}\left(f_{\mathrm{CO}}\right)= & 2.33( \pm 3 \%) \mathrm{mV}, \\
& \text { gradient } m=-2.43( \pm 6 \%)
\end{aligned}
$$

perturbed system: $f_{\mathrm{CO}}=7.41( \pm 2 \%) \mathrm{sccm}$,

$$
\text { fixed point } \begin{aligned}
x_{\mathrm{s}}\left(f_{\mathrm{CO}}\right)= & 2.22( \pm 3 \%) \mathrm{mV}, \\
& \text { gradient } m=-2.46( \pm 6 \%)
\end{aligned}
$$

yielding

$$
g=-0.24( \pm 125 \%) \mathrm{mV} \mathrm{sccm}^{-1}
$$

for the current system. This value is used as a first estimate of the control parameter. The large experimental uncertainty does, however, require that the operator be allowed to "optimize" the choice of $g$ during the actual control experiment, so a facility for this is incorporated into the control software. We may also note here that the gradient $m$ appears to satisfy the requirement of the basic SPF method in that is not changed by the perturbation.

During an experiment, then, once the appropriate value for the control parameter has been set, the maxima in the emitted light intensity are monitored by the control algorithm and once a point on the map is observed to fall within some predetermined region in the vicinity of the unstable period- 1 fixed point (typically we choose that $x_{n}$ should lie within $\pm 25 \%$ of $x_{\mathrm{F}}$ estimated from the map), the control algorithm is activated automatically and the $\mathrm{CO}$ flow rate is perturbed according to eq 7. Further refinement of the choice of the control parameter may be made if the convergence to a controlled period-1 state is seen to fail or if the calculated perturbation to the flow rate would be so large as to move the system out of the chaotic region in terms of the operating conditions.

The successful control of a period-1 state within the chaotic region for the $\mathrm{CO}+\mathrm{O}_{2}$ reaction under the above experimental conditions using the SPF method is illustrated in Figure 3A,B. In Figure 3A, the time series from the PMT record is shown, exhibiting a chaotic response up to the point at which the control algorithm is activated and then evolution to a period of control. At the end of the control period (decided by the operator), the system returns to the chaotic state. Sequences of up to 60 controlled period-1 ignitions have been achieved with care through this approach. The convergence of the maxima to the controlled period-1 state is shown in Figure 3B: there is a definite "transient period" between the onset of control and the 

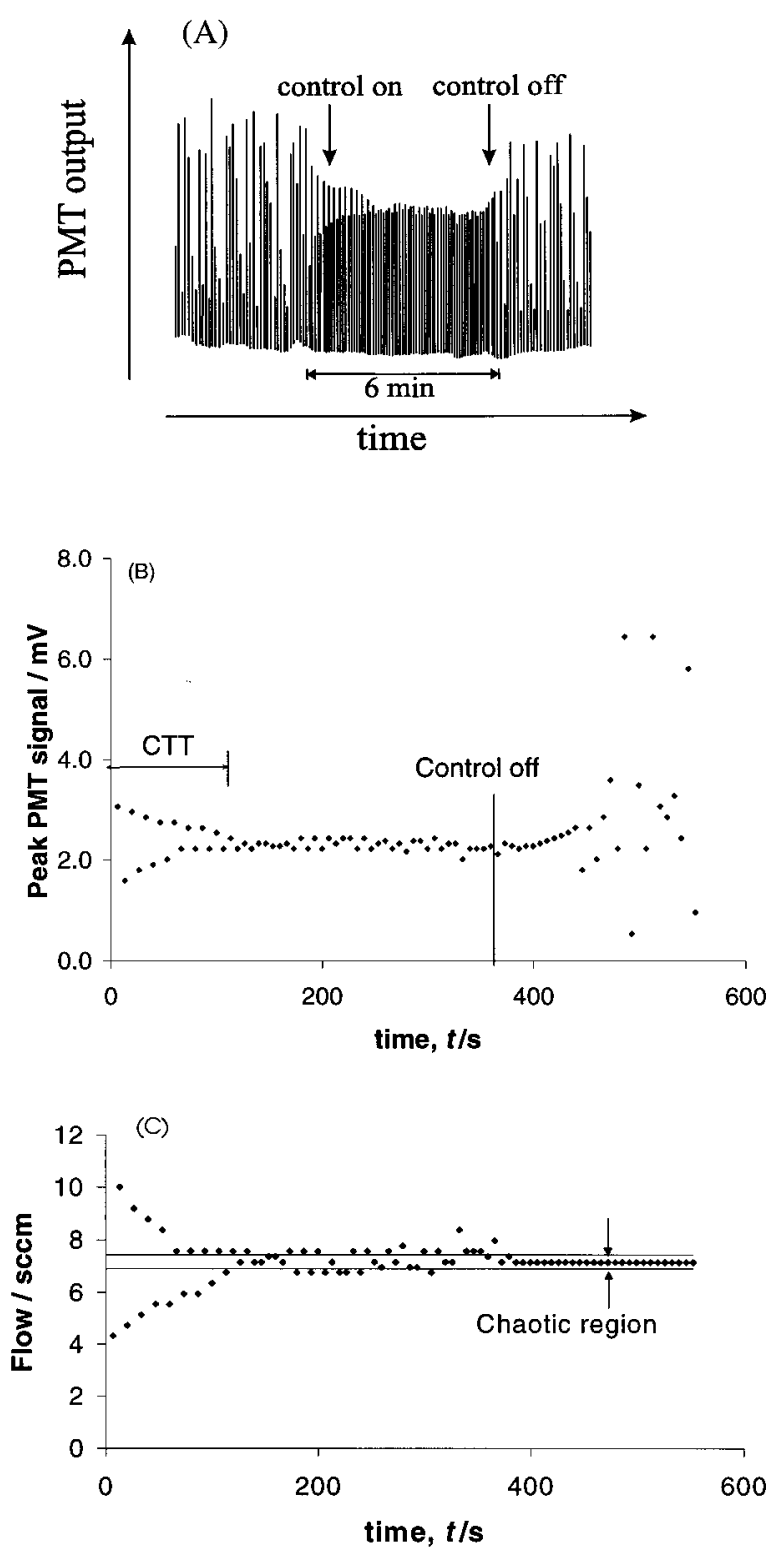

Figure 3. Control of chaos by SPF algorithm: (A) time series from PMT showing approach to stabilized period-1 orbit after control algorithm is activated ("control on") and return to chaotic state when control algorithm is deactivated ("control off"); (B) plot of observed maximum PMT signal from time series during and after control, showing the "control transient time" (CTT); (C) variation of CO flow rate due to perturbations imposed by control algorithm. The horizontal lines indicate the upper and lower limits for the $\mathrm{CO}$ flow for which the unperturbed system exhibits the chaotic response.

establishment of a period-1 response. For the system shown in Figure 3, this "control transient time" (CTT) period is $113 \mathrm{~s}$. (We should note that the CTT as defined here differs from the "transient time" introduced by Ott et al. ${ }^{9}$ and used by Kiss et al. ${ }^{18}$ which refers to the time taken by the system to approach the neighborhood of the fixed point: in that work the SPF method effectively produces control in one step once activated.)

The variation in the $\mathrm{CO}$ flow rate imposed on the system by the control algorithm is plotted in Figure 3C. The initial perturbations take the system beyond the upper limit of spontaneous chaos, but soon the perturbation settles to a small value so that the system lies within the range of spontaneous chaos. It is noticeable, however, for this reaction that occasional large perturbations are required which again take the system transiently outside the spontaneous chaos range. (d) Variation of Perturbation Time. In the experiments described in the previous section, the control perturbation is applied immediately after the calculation of eq 7 is completed and the perturbed flow rate is maintained over the whole period until the next maximum is observed. A new perturbation is then calculated and this is applied over the whole period until the next maximum, and so on. In this section, we describe the effect of varying the fraction of the ignition period over which the perturbation is applied. Before the control algorithm is activated, the system is monitored as described previously in its chaotic state. We now also determine the mean period $\langle\tau\rangle$ between ignitions during this monitoring phase. Typically, we find $\langle\tau\rangle$ $=6.6 \mathrm{~s}$. (A feature of the $\mathrm{CO}+\mathrm{O}_{2}$ system is that the period between ignitions does not vary significantly even though the amplitudes vary markedly during chaotic evolution.) The control algorithm can now be implemented but with the perturbation applied for only some fraction of the ignition period, with the flow rate then returned to its unperturbed value until the next maximum is observed. Control is observed for all cases with the perturbation applied for between $10 \%$ and $100 \%$ of a given ignition period using the same value for the control parameter $g$ in each case. The transient evolution from the chaotic to the controlled response following the activation of the control algorithm does, however, vary significantly with this parameter, as indicated in Figure 4.

Initially, decreasing the period for which the control perturbation is applied leads to an increased rate at which the control is established. The rate of establishment of control appears to attain some plateau for perturbations applied for between $20 \%$ and $30 \%$ of the ignition period, as indicated in Figure 5, which plots the inverse of the CTT determined from the traces in Figure 4. Control is not possible in this reaction system for perturbations applied for less than $20 \%$ of the oscillatory period, perhaps due to the finite response time of the mass flow controllers in adjusting to their new values.

(e) Drift in the Next-Maximum Map. Despite the success of the modified SPF algorithm, the $\mathrm{CO}+\mathrm{O}_{2}$ system exhibits a number of features that make control less than straightforward compared to some other experimental systems. A major difficulty for long-term control arises through the tendency of the reaction to "drift" over the course of an experimental run. Thus, the boundaries separating different oscillatory modes in the $p-T_{\mathrm{a}}$ diagram, and even the ignition limit itself, change during the course of an experiment-almost certainly due to the continual slow conditioning of the reactor surface. To illustrate the effect of this for the SPF control algorithm, the variation of the nextmaximum map in time over the course of $1 \mathrm{~h}$ for an experiment under constant experimental conditions is shown in Figure 6A. A considerable drift in the map, and hence in the fixed point, occurs even under these "constant" conditions, so small imposed perturbations calculated on the basis of eq 7 could easily be swamped. This effect can be reduced by allowing the system a long conditioning period before any attempt at control is implemented, but is never completely removed. Similar, but longer time scale drifts are observed in other systems for which control has been achieved, most notably the control of chaos in electrodissolution reactions. ${ }^{18}$

Long-lived effects on the evolution of the system can also arise simply from the imposition of a perturbation on the flow rate. The next-maximum maps recorded for identical operating conditions before and $60 \mathrm{~s}$ after a relatively large perturbation (1.0 sccm on the $\mathrm{O}_{2}$ flow rate applied for $300 \mathrm{~s}$ and then removed) are shown in Figure 6B. The map clearly remains significantly shifted even after the perturbation has been 

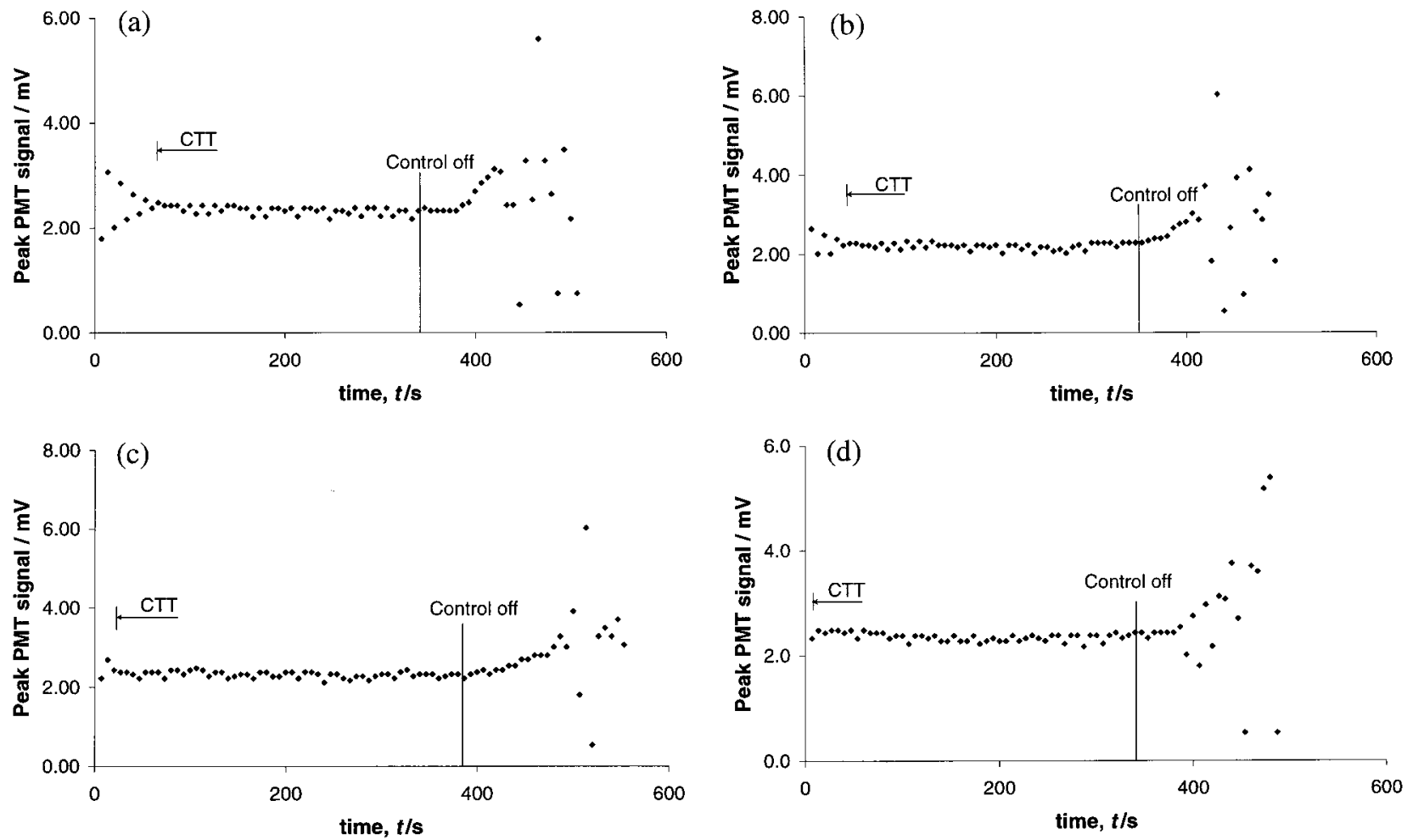

Figure 4. Plots of observed maximum PMT signal from time series for modified SPF method with perturbation applied for only a part of the oscillatory period showing effect on CTT: (a) perturbations applied for $80 \%$ of period; (b) perturbations applied for $60 \%$ of period; (c) perturbations applied for $40 \%$ of period; (d) perturbations applied for $20 \%$ of period.

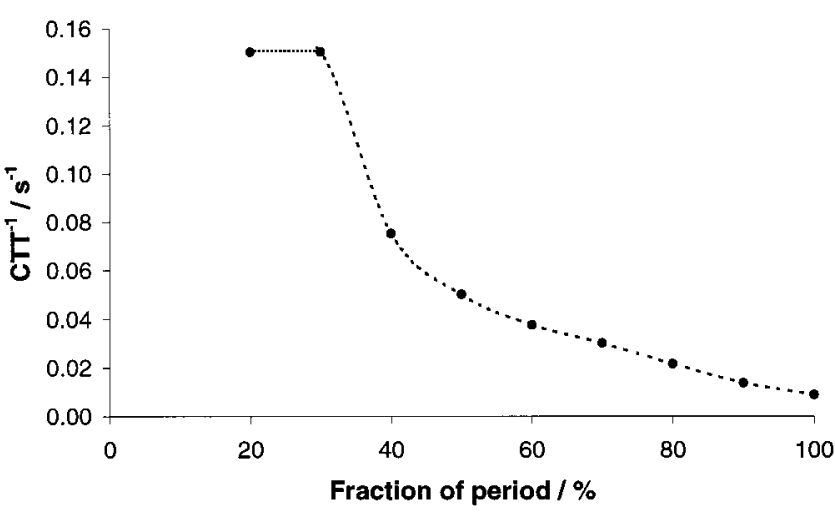

Figure 5. Variation of the inverse control transient time (CTT) $)^{-1}$ with the fraction of the oscillatory period for which the perturbation is applied. A large value indicates faster convergence to the controlled period-1 state. The solid line is draw to guide the eye and suggests an optimum at ca. $20-30 \%$. Control is not achieved for perturbations applied for less than $20 \%$ of the oscillatory period.

removed. This effect means that care must be taken to allow only small-amplitude perturbations in the control algorithm.

\section{Numerical Study of $\mathbf{H}_{2}+\mathrm{O}_{2}$ Reaction}

The operational points described at the end of the previous section indicate that descriptive quantitative measures of the control, beyond those presented in Figure 5, are effectively unavailable from the experimental system. A satisfactory chemical kinetic mechanism describing the chaotic behavior observed experimentally in the $\mathrm{CO}+\mathrm{O}_{2}$ system does not yet exist, but such a mechanism for similar responses in the related $\mathrm{H}_{2}+\mathrm{O}_{2}$ reaction has been established. ${ }^{22}$ In this section, we report on a numerical study of chaos control in this model.

The "minimal complex oscillator model" for the $\mathrm{H}_{2}+\mathrm{O}_{2}$ system comprises 12 elementary reactions involving seven chemical species and the reacting gas temperature. Numerical integration of the corresponding reaction rate and energy balance equations for this reaction in a CSTR have been performed using the SPRINT algorithm, which is appropriate for stiff systems and allows for the temperature dependence of reaction heat capacities and enthalpies. With a molar inflow ratio of the reactants $\mathrm{H}_{2}: \mathrm{O}_{2}=7: 3$, the system exhibits chaotic oscillations at a total pressure of $10 \mathrm{mmHg}$ with $t_{\mathrm{res}}=1.4 \mathrm{~s}$ and $T_{\mathrm{a}}=702.7$ $\mathrm{K}$. For the present purposes, guided by the experiments described above, the bifurcation parameter $p$ adopted will be the ratio of the inflow concentrations (with the total molar inflow concentration being held fixed). We take $p=0$ to correspond to the above ratio, while $p=1$ corresponds to a system with a $1 \%$ increase in the $\mathrm{H}_{2}$ inflow and the $\mathrm{O}_{2}$ decreased correspondingly (giving $\mathrm{H}_{2}: \mathrm{O}_{2}=7.1: 2.9$ ). Complex (chaotic) behavior is observed over the range $-6<p<1.7$ approximately. The nextreturn map based on the maximum temperature during the oscillation for $p=0$ is shown in Figure 7. The seven variable model exhibits a nearly one-dimensional next-return map, with a fixed point $x_{\mathrm{F}}=1314.0$ and a slope $m=-2.39$ at the fixed point. Different control strategies have been implemented to stabilize this fixed point.

(a) SPF Control. Here we seek to determine appropriate perturbations $\delta p$ of the form

$$
\delta p=K \delta x_{n}
$$

with $K=m /(1-m)\left(\mathrm{d} x_{\mathrm{F}} / \mathrm{d} p\right)=1 / g$ (see eq 8). Recalculating the next-return map for $p=0.08$, we find $x_{\mathrm{F}}=1310.5$ and $m$ $=-2.46$, yielding $g=-62.0$ and $K=-1.6 \times 10^{-2}$. Attempts to stabilize the period-1 fixed point using the SPF method with the perturbation applied for the whole oscillatory period failed in our numerical integrations with this system.

(b) Higher-Dimensional Control Method. A general method for control extending the SPF method to either involve perturba- 

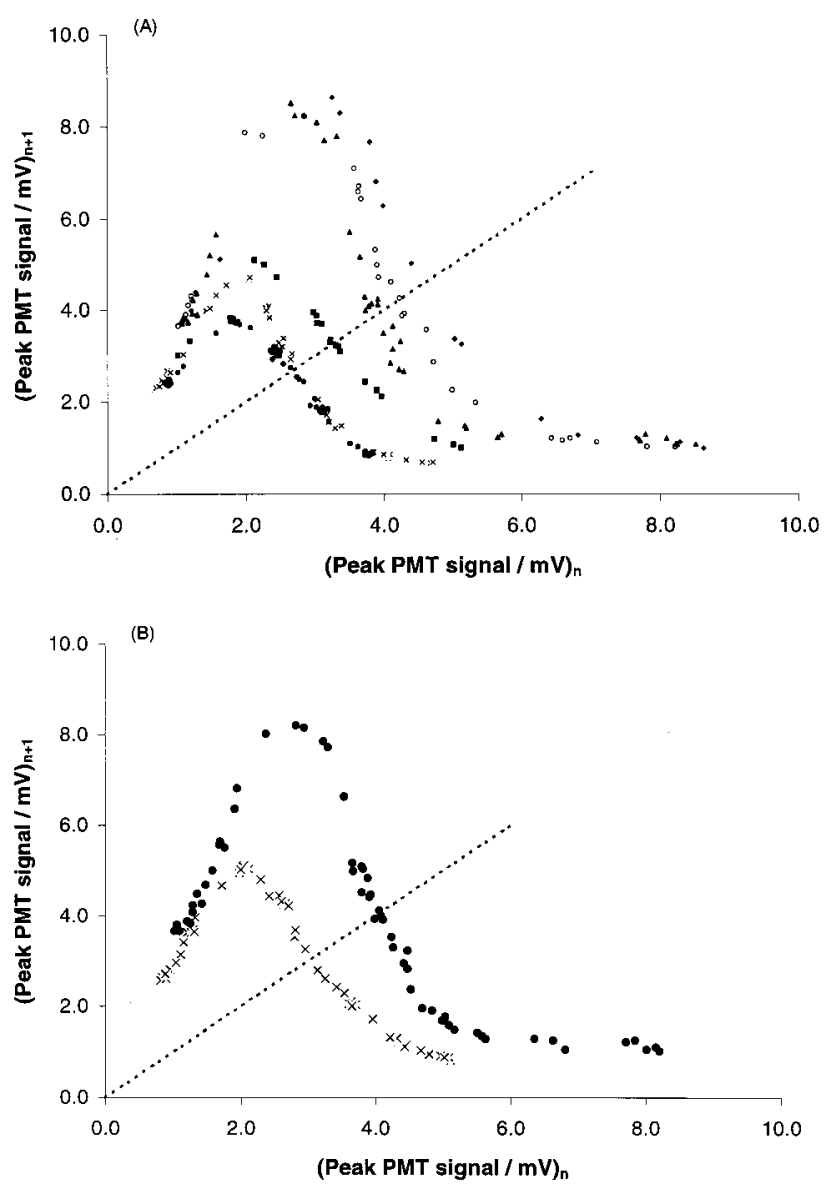

Figure 6. Evidence of drift in the next-return map for the $\mathrm{CO}+\mathrm{O}_{2}$ system. (A) Evolution of the next-maximum map in time for an experiment under constant experimental conditions: data collected at the following times: $\bullet, 5 \mathrm{~min} ; \bigcirc, 10 \mathrm{~min} ; \mathbf{\Delta}, 20 \mathrm{~min} ; \mathbf{\square}, 30 \mathrm{~min} ; \times$, $40 \mathrm{~min}$; $60 \mathrm{~min}$. (B) Shift in map following imposition and then removal of a large perturbation to the $\mathrm{CO}$ flow: $\times$, before perturbation; -, after perturbation.

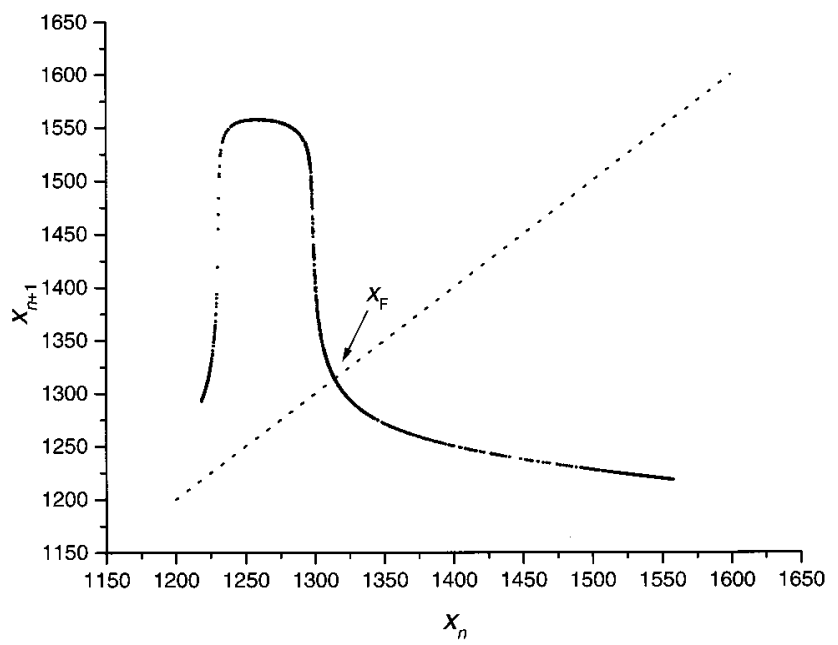

Figure 7. The next-return map based on the maximum temperature for the unperturbed $\mathrm{H}_{2}+\mathrm{O}_{2}$ system.

tion of several parameters or to exploit successive perturbations of a single parameter has been proposed by Petrov et al. ${ }^{23}$ Here, we apply this method to explain the failure of the SPF algorithm in the present case. The extended method interrogates the evolution of the system in the vicinity of the fixed point subject to a random sequence of parameter perturbations. The observed
TABLE 2: Variation of Fitting Error with Dimension of Linear Model (Eq 9) at $p=0$ with Perturbation Applied for Different Durations

\begin{tabular}{|c|c|c|c|c|c|}
\hline \multicolumn{6}{|c|}{ perturbation duration } \\
\hline \multicolumn{2}{|c|}{ full cycle $(1.5 \mathrm{~s})$} & \multicolumn{2}{|c|}{$0.3 \mathrm{~s}$} & \multicolumn{2}{|c|}{$0.5 \mathrm{~s}$} \\
\hline$N$ & $\sigma$ & $N$ & $\sigma$ & $N$ & $\sigma$ \\
\hline 1 & 2.30 & 1 & 0.33 & 1 & 0.39 \\
\hline 2 & 0.25 & 2 & 0.24 & 2 & 0.31 \\
\hline 3 & 0.17 & 3 & 0.25 & 3 & 0.22 \\
\hline
\end{tabular}

response map is then fitted to the following expansion

$$
\delta x_{n+1}=\sum_{k=1}^{N} a_{N-k+1} \delta x_{n-k+1}+\sum_{k=1}^{N} b_{N-k+1} \delta p_{n-k+1}
$$

where $N$ is a "fitting dimension" of the system. The eigenvalues of the controlled system $\lambda_{1}, \ldots, \lambda_{N+1}$ can then be set to any desired values (the so-called "target dynamics", e.g., $\lambda_{i}=0$ for all $i$ for so-called "dead-beat control") according to the control formula

$$
\delta p_{n}=\sum_{k=1}^{N} q_{N-k+1} \delta x_{n-k+1}+\sum_{k=2}^{N} r_{N-k+1} \delta p_{n-k+1}
$$

where the control coefficients $q_{i}$ and $r_{i}$ are determined as described in Petrov et al. ${ }^{24}$

The $\mathrm{H}_{2}+\mathrm{O}_{2}$ model was integrated numerically subject to random perturbations in the range $\delta p=[-0.05,0.05]$. Typically the integrations were continued for approximately 12000 oscillations, providing approximately 40 points in the vicinity of the unstable period-1 fixed point of the next-maximum map. These points were then fitted to the appropriate form of eq 10 and the fitting error $\sigma$ determined as a function of dimension $N$. The results are shown in Table 2.

The large fitting error for $N=1$ indicates that the onedimensional model cannot be fitted to the perturbed system dynamics satisfactorily, and hence the SPF algorithm fails. Although the dynamics of the unperturbed system is approximately one-dimensional, the perturbation continuously diverts the trajectory away from the attractor, resulting in a higher dimension for the controlled system.

With $N=2$, using $\lambda_{1}=\lambda_{2}=\lambda_{3}=0$ as the target dynamics, the control formula becomes

$\delta p_{n}=8.2 \times 10^{-2} \delta x_{n}+7.2 \times 10^{-3} \delta x_{n-1}+4.6 \delta p_{n-1}$

This linear model is only expected to work in the linear region close to the fixed point: if the algorithm is applied outside this range, the system is likely to diverge from the fixed point. In our calculations we imposed a maximum allowed perturbation $\left|\delta p_{\max }\right|=0.5$. Subject to this constraint, the chaotic response has been successfully controlled via eq 12, as indicated in Figure 8. The controlled system and the imposed perturbation are, however, somewhat "noisy" and the mean value of the control parameter during the control period is clearly somewhat lower than zero; i.e., the operating conditions have been varied from the autonomous system (although it remains well within the chaotic range).

The nature of this response may arise from the relatively large value of the third control parameter (the coefficient of the $\delta p_{n-1}$ term) in eq 12. In the presence of real experimental noise an alternative approach may be required. In other systems, a recursive proportional algorithm has been exploited ${ }^{16}$ (neglecting the term involving $\delta x_{n-1}$ in eq 12). Petrov et al. applied 
a)

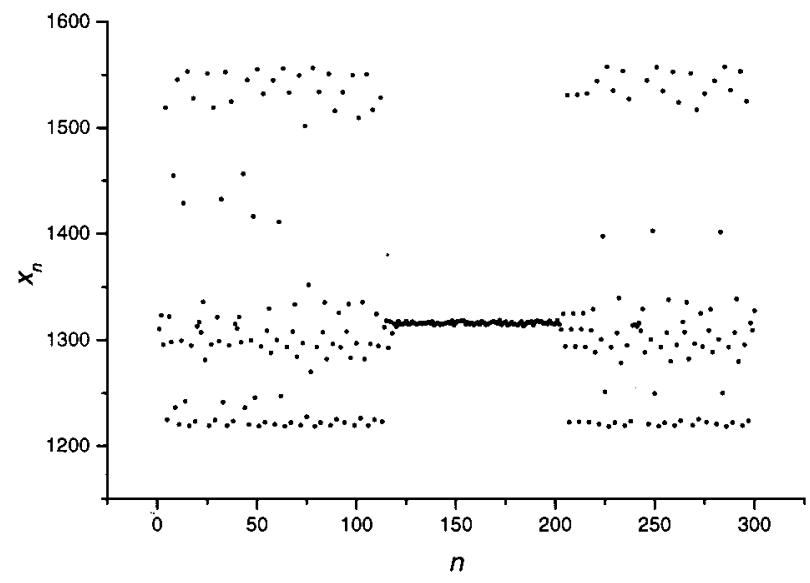

b)

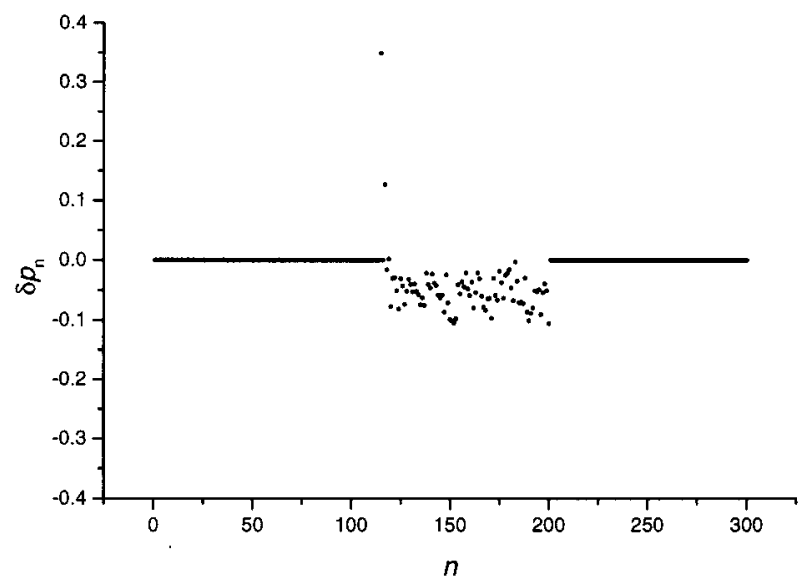

Figure 8. (a) Plot of observed temperature maxima for $\mathrm{H}_{2}+\mathrm{O}_{2}$ system using the control eq 12 activated at oscillation number $n=100$. The control becomes effective from $n \approx 110$ when $x_{n}$ reaches the vicinity of the fixed point. (b) The perturbation $\delta p_{n}$ applied during the control procedure.

perturbations to two control parameters: one parameter was used to target the fixed point and the second was varied to keep the phase point on the attractor. The simultaneous variation of two different experimental parameters is somewhat demanding in the combustion reactions of interest here: different control devices (e.g., for the mass flow and the oven temperature) are typically subject to differing time constants and a substantially larger set of data is required to characterize the map and the effect of two parameter variations. Carr and Schwartz ${ }^{25}$ have shown that the length of time for which a perturbation is applied can be used as a second parameter. We now investigate this approach with the present numerical model.

(c) Perturbation Applied for $\mathbf{0 . 3}$ s. The fitting errors arising for systems with $N=1,2$, and 3 for which the perturbation is applied for only $0.3 \mathrm{~s}$ between each maximum (approximately $20 \%$ of the period of the unstable period- 1 oscillation) are also given in Table 2. In this case the system can be regarded as one-dimensional even in the presence of random perturbations. Robust control is exhibited with the control formula

$$
\delta p_{n}=-4.7 \times 10^{-2} x_{n}
$$

as indicated in Figure 9. The fixed point is successfully stabilized, and the perturbations to the flow ratio are now smaller and more evenly distributed about $p=0$. The SPF method is

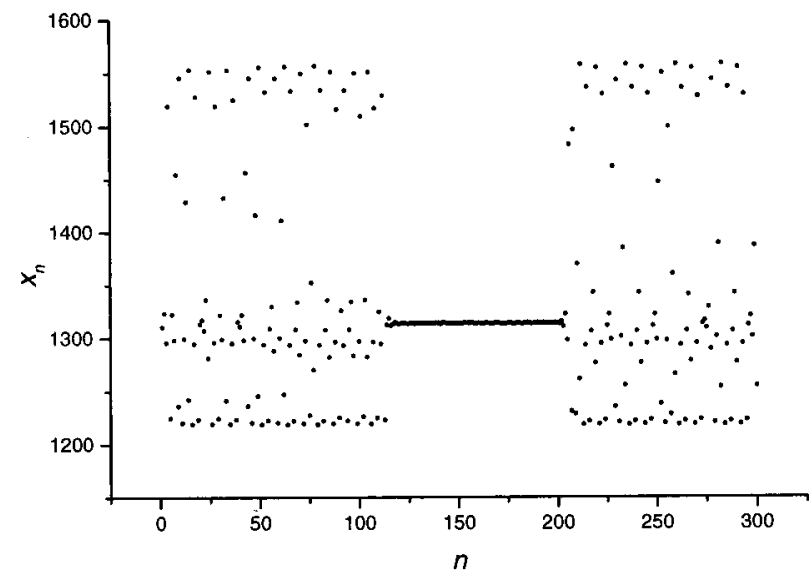

b)

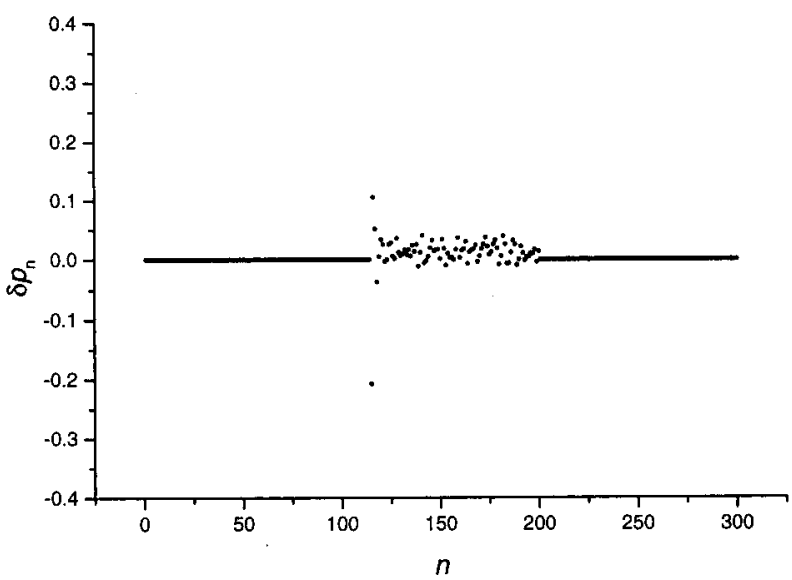

Figure 9. (a) Plot of observed temperature maxima for $\mathrm{H}_{2}+\mathrm{O}_{2}$ system using the modified SPF control algorithm, with the perturbation applied for only $0.3 \mathrm{~s}$ in each oscillation. (b) The perturbation $\delta p_{n}$ applied during the control procedure. Control is switched off at $n=200$.

therefore expected to work in this case and is observed in our computations using $K=-7.1 \times 10^{-2}$, corresponding to $g=$ -14 .1. (Note that the control constants obtained with the SPF and random perturbation algorithms are numerically different although of the same magnitude.)

(d) Perturbation Applied for 0.5 s. The fitting errors in Table 2 for this case are comparable for those with the perturbation applied for $0.3 \mathrm{~s}$, so control might be expected even with $N=1$. However, as illustrated in Figure 10, the control formula $\delta p_{n}=-8.3 \times 10^{-2} \delta x_{n}$ obtained in this case does not give robust control. The amplitude is brought to the neighborhood of the fixed point for several iterations, but then diverges. Similarly, the SPF method, with $K=-7.1 \times 10^{-2}$ so $g=$ -14.1 , also fails. Control is successful with $N=2$, with $\delta p_{n}=$ $-1.12 \times 10^{-1} \delta x_{n}-7.8 \times 10^{-4} \delta x_{n-1}-0.43 \delta p_{n-1}$.

\section{Some Comments on the SPF Method}

In this section we investigate the application of the SPF method for systems with underlying higher-dimensional dynamics such as the $\mathrm{CO}+\mathrm{O}_{2}$ or $\mathrm{H}_{2}+\mathrm{O}_{2}$ systems studied here. In particular, we will try to determine the conditions under which the modification of applying perturbations for a limited duration will be effective in a given instance and what determines the optimum perturbation duration.

We have integrated the governing equations for the $\mathrm{H}_{2}+\mathrm{O}_{2}$ model under spontaneously chaotic conditions. The response 


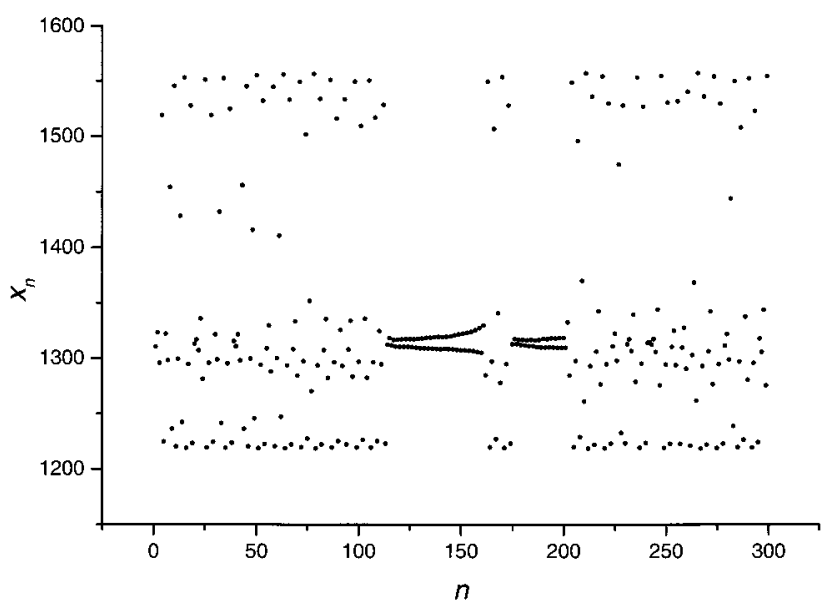

Figure 10. Failure of one-dimensional control algorithm with perturbation applied for $0.5 \mathrm{~s}$.

has then been interrogated through the application of random perturbations as above, but now with the perturbations applied for only a fixed duration in each cycle, less than the full oscillatory period. Again, approximate 40 points lying within the vicinity of the unstable period-1 fixed point were collected in each case, and these were fitted to the two-dimensional fitting equation in the form

$$
\delta x_{n+1}=a_{1} \delta x_{n}+a_{2} \delta x_{n-1}+b_{1} \delta p_{n}+b_{2} \delta p_{n-1}
$$

to yield the appropriate values of the fitting coefficients $a_{i}, b_{i}$ for a given perturbation duration. Next, we imagine that an SPF algorithm is to be implemented, so that $\delta p_{n}$ is related to $\delta x_{n}$ by $\delta p_{n}=K \delta x_{n}$ (see eq 9) and $\delta p_{n-1}$ will be similarly related to $\delta x_{n-1}$. Substituting these forms into eq 13, we obtain

$$
\delta x_{n+1}=\left(a_{1}+b_{1} K\right) \delta x_{n}+\left(a_{2}+b_{2} K\right) \delta x_{n-1}
$$

This equation can be used to determine the fitting eigenvalues $\lambda_{1,2}$ : introducing a dummy variable $\delta y_{n}=\delta x_{n-1}$, the map can be written in the form

$$
\begin{gathered}
\delta x_{n+1}=\left(a_{1}+b_{1} K\right) \delta x_{n}+\left(a_{2}+b_{2} K\right) \delta y_{n} \\
\delta y_{n+1}=\delta x_{n}
\end{gathered}
$$
$\mathbf{J}$

The eigenvalues are then determined from the Jacobian matrix

$$
\mathbf{J}=\left(\begin{array}{cc}
a_{1}+b_{1} K & a_{2}+b_{2} K \\
1 & 0
\end{array}\right)
$$

yielding the characteristic equation

$$
\lambda^{2}-\left(a_{1}+b_{1} K\right) \lambda-\left(a_{2}+b_{2} K\right)=0
$$

A number of points concerning this equation can be made. For the unperturbed system, $b_{i}=0$ and the roots of $\lambda^{2}-a_{1} \lambda$ $-a_{2}=0$ yield the eigenvalues of the two-dimensional map for the autonomous system.

The SPF method based on a one-dimensional map has $a_{2}=$ $b_{2}=0$, yielding

$$
\lambda_{\mathrm{SPF}}=\left(a_{1}+b_{1} K\right), \quad 0
$$

For the target dynamics for the SPF method, we then choose the value of $K=-a_{1} / b_{1}$ so the first root becomes zero. The higher-dimensional character of the system is thus reflected in a

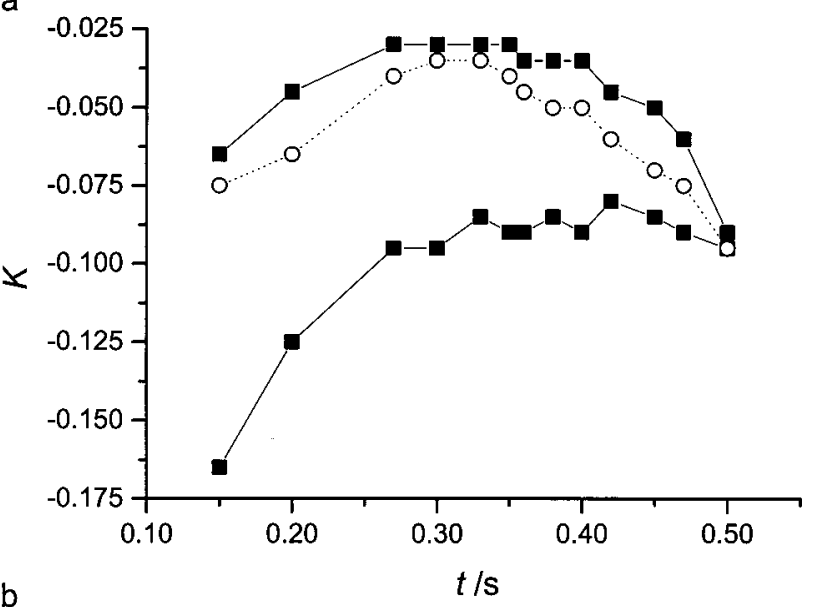

b

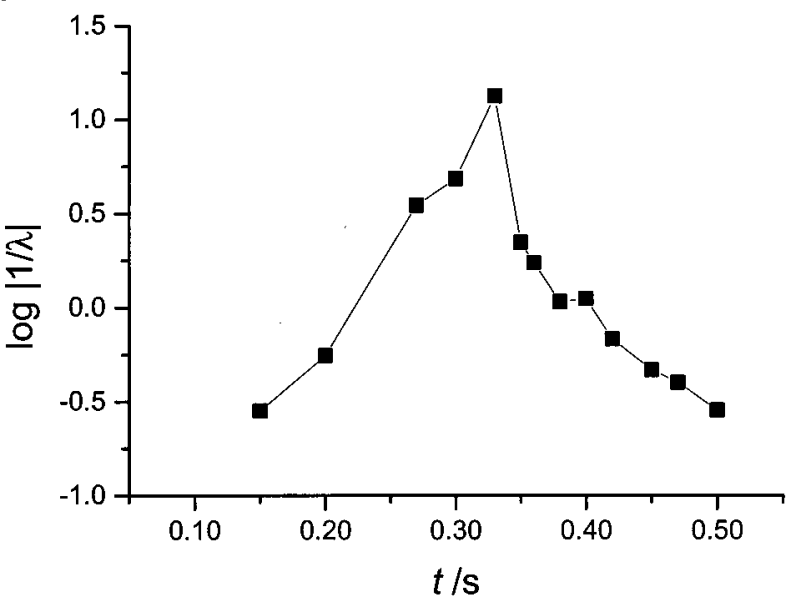

Figure 11. (a) Plot of the available "control space" for the $\mathrm{H}_{2}+\mathrm{O}_{2}$ model system. Control is only achievable for combinations of the control parameter $K$ and the perturbation duration $t_{\mathrm{p}}$ between the two solid curves. (b) Variation of the control transient time, represented as log$(1 /|\lambda|)$ with perturbation time $t$ for a system with $K=-0.035$ showing a pronounced optimum choice of perturbation period.

the changes in the eigenvalues from $\lambda_{\mathrm{SPF}}$ that arise due to the final term in eq 15, and as this becomes significant, the SPF choice for $K$ will become inappropriate and the method will fail. (This presumably is part of the reason that the practical implementation of the SPF method to the $\mathrm{CO}+\mathrm{O}_{2}$ system described in section 3 and for other systems elsewhere typically requires the operator to vary the control parameter from that calculated on the basis of the SPF algorithm.)

Suppose we make a particular choice for the control parameter $K$ (perhaps the corresponding SPF value). Then the eigenvalues are given by

$$
\lambda_{1,2}=(1 / 2)\left\{a_{1}+b_{1} K \pm\left[\left(a_{1}+b_{1} K\right)^{2}+4\left(a_{2}+b_{2} K\right)\right]^{1 / 2}\right.
$$

Iterations of the control algorithm with approach/diverge from the period-1 fixed point according to whether the magnitudes $\left|\lambda_{1,2}\right|$ are less than or greater than 1 . For the control algorithm to work, we require both eigenvalues to have magnitude less than unity. This effectively imposes a constraint on the range of suitable choices for the control parameter $K$ in terms of the fitting parameters $a_{i}$ and $b_{i}$. The range of acceptable values for $K$ for the $\mathrm{H}_{2}+\mathrm{O}_{2}$ system is shown as a function of the duration of the imposed perturbation in Figure 11a. Control using the modified SPF algorithm is only possible within the closed region in the diagram corresponding to $\left|\lambda_{1,2}\right|<1$. 
In Figure 11b, we plot the inverse of the logarithm of the principal eigenvalue (that with magnitude closer to unity) as a function of the perturbation duration for a system with $K=$ -0.035 . This is qualitatively equivalent to the plot of the inverse control transient time $\mathrm{CTT}^{-1}$ given in Figure 5 for the experimental $\mathrm{CO}+\mathrm{O}_{2}$ system. Control is only possible with $\log |1 / \lambda|>0$, and hence, for this value of $K$ it is only possible for a perturbation time $t_{\mathrm{p}}$ in the range $0.22 \mathrm{~s}<t_{\mathrm{p}}<0.41 \mathrm{~s}$. The plot shows a distinct maximum corresponding to an optimum choice for the perturbation duration in a manner similar to that observed in the experiments. For the present choice of $K$, the optimal perturbation time is ca. $0.33 \mathrm{~s}$. We have plotted the "optimal" value of $t_{\mathrm{p}}$ as a function of the control parameter $K$ in Figure 11a. An interesting point is that, for some choices for $K$, there appear to be two values for this locus, indicating that the corresponding $\log (1 /|\lambda|)-t_{\mathrm{p}}$ graph may show more than one maximum. This point will be investigated elsewhere.

\section{Conclusions}

The present work has demonstrated the application of the SPF method in general terms to the control of chaos in a technologically important combustion reaction. The method does not require any detailed knowledge of the chemical mechanism driving the reaction, nor does it involve large perturbations that take the system outside the region of chaotic behavior. All the information required to implement the control algorithm is determined experimentally: this involves the location of the fixed point of the next-maximum map, the slope of the map in the vicinity of this fixed point, and the shift in the fixed point accompanying a small change in one of the experimental control parameters. The methodology also works even though the present system shows a marked "drift" over the course of the experiment. We find that there is a considerable improvement in the efficiency of the control algorithm in the present case if the perturbation is applied only for a fraction of the oscillatory period. The numerical study of the $\mathrm{H}_{2}+\mathrm{O}_{2}$ system also demonstrates this feature and suggests that it arises because the perturbations displace the system significantly from the original attractor. As noted by Petrov et al., ${ }^{23}$ in such cases the behavior of the perturbed system is partly controlled by the stable manifolds that are not described by the one-dimensional map of the unperturbed system. This explains why a higherdimensional algorithm is required. The specific implementation of the higher-dimensional control exploited in the experiments here, treating the period for which the perturbation is applied as a parameter, differs from that used by Carr and Schwartz. ${ }^{25}$ In their numerical computations, the duration of the perturbation was varied from oscillation to oscillation in an appropriate manner determined from the control equation. In our approach, we choose a fixed duration for which the perturbation is applied in each oscillation. This simplification makes the control algorithm substantially easier to apply in practice and, subject to some empirical optimization, still appears to work satisfactorily.

Acknowledgment. This work was supported by the British Council and the Hungarian Scholarship Board (Ministry of Education) under the Joint Academic Research Programme scheme (Project No. 010), by the ESF Scientific Programme REACTOR, by the EPSRC (M.L.D., M.R.T.), and by following Hungarian Research Grants: OTKA T025375 and FKFP 0455/ 1997.

\section{References and Notes}

(1) Lewis, B.; von Elbe, G. Combustion, flames and explosions of gases; Academic Press: Orlando, 1987.

(2) Ashmore, P. G.; Norrish, R. G. W. Nature 1951, 167, 390.

(3) Linnett, J. W.; Reuben, B. G.; Wheatley, T. F. Combust. Flame 1968, 12,325 .

(4) Bond, J. R.; Gray, P.; Griffiths, J. F. Proc. R. Soc. London, A 1981 A375, 43 .

(5) Bond, J. R.; Gray, P.; Griffiths, J. F.; Scott, S. K. Proc. R. Soc. London, A 1982, A381, 293.

(6) Gray, P.; Griffiths, J. F.; Scott, S. K. Proc. R. Soc. London, A 1985, A397, 21.

(7) Johnson, B. R.; Scott, S. K. J. Chem. Soc., Faraday Trans. 1990, 86,3701 .

(8) Johnson, B. R.; Griffiths, J. F.; Scott, S. K. Chaos 1991, 1, 387.

(9) Ott, E.; Grebogi, C.; Yorke, J. A. Phys. Rev. Lett. 1990, 64, 1196.

(10) Peng, B.; Petrov, V.; Showalter, K. J. Phys. Chem. 1991, 95, 4597.

(11) Petrov, V.; Gáspár, V.; Masere, J.; Showalter, K. Nature 1993, 361, 240 .

(12) Pyragas, K. Phys. Lett. A 1992, 170, 421.

(13) Hunt, E. R. Phys. Rev. Lett. 1991, 67, 1953.

(14) Schneider, F. W.; Blittersdorf, R.; Förster, A.; Hauck, T.; Lebender, D.; Mülller, J. J. Phys. Chem. 1993, 97, 12244.

(15) Lekebusch, A.; Förster, A.; Schneider, F. W. J. Phys. Chem. 1995 99,681 .

(16) Rhode, M. A.; Rollins, R. W.; Dewald, H. D. Chaos 1997, 7, 653.

(17) Parmananda, P.; Sherard, P.; Rollins, R. W.; Dewald, H. D. Phys. Rev. E 1993, 47, R3003.

(18) Kiss, I. Z.; Gáspár, V.; Nyikos, L.; Parmananda, P. J. Phys. Chem. A 1997, 101, 8668 .

(19) Parmananda, P.; Madrigal, R.; Rivera, M.; Nyikos, L.; Kiss, I. Z.; Gáspár, V. Phys. Rev. E 1999, 59, 5266.

(20) Focus issue on control and synchronization of chaos. Chaos 1997, 7 (4) 509-687.

(21) Boccaletti, S.; Grebogi, C.; Lai, Y.-C.; Mancini, H.; Maza, D. Phys. Rep. 2000, 329, 103.

(22) Johnson, B. R.; Scott, S. K. J. Chem. Soc., Faraday Trans. 1997, 93, 2997.

(23) Petrov, V.; Peng, B.; Showalter, K. J. Chem. Phys. 1992, 96, 7506.

(24) Petrov, V.; Mihaliuk, E.; Scott, S. K.; Showalter, K. Phys. Rev. E 1995, 51, 3988 .

(25) Carr, T. W.; Schwartz, I. B. Phys. Rev. E 1994, 50, 3410. 